\title{
Neutrino-related questions in nuclear and astro-particle physics
}

\section{Osvaldo Civitarese *}

Department of Physics, University of La Plata, La Plata, Argentina

\section{Edited by:}

Frank Franz Deppisch, University

College London, UK

\section{Reviewed by:}

Janusz Gluza, University of Silesia, Poland

Michael Andreas Schmidt, The

University of Melbourne, Australia

*Correspondence:

Osvaldo Civitarese, Department of

Physics, University of La Plata

c.c. 67 (1900), La Plata, Argentina

e-mail:osvaldo.civitarese@

fisica.unlp.edu.ar

\section{INTRODUCTION}

The frontiers in nuclear, particle and astro-particle physics are delimited by a line of challenging questions, particularly those related to neutrino physics (neutrino oscillations and neutrino mass), and its consequences upon the abundances of primordial elements in Big Bang Nucleosynthesis (BBN), the physics of supernovae (SN), and the observation of highly energetic phenomena (neutrino emission from quasars and microquasars).

The suitable theoretical tools which are available, at the time of performing actual calculations in these various scenarios, include:

- Models of the neutrino: that is oscillation parameters, mixing with sterile neutrinos and the number of sterile neutrino species, mass ordering and hierarchies.

- Models of nuclear structure at low energies, which are needed to compute reliable nuclear matrix elements for rare decays, like the neutrinoless double beta decay, and cross sections for neutrino-nucleus scattering, and for the calculation of $\mathrm{BBN}$ processes.

- Relativistic models of nuclear structure, for the scattering neutrino-nucleus at high energies, and for the calculation of neutrino related astrophysical events, like quasars and microquasars.

Naturally, one should also consider astrophysical models of highly energetic processes which generate neutrinos in the field of star models, as well as in cosmology.

In this inaugural letter, I shall try to give a flavor of the sort of questions and motivations which the Journal will welcome. I shall refer to some of the open problems, which I think may have high impact on the field, a choice which obviously represents a small sample of the complete universe of today's problems.

\section{SOME OPEN PROBLEMS}

Our short list includes the following topics. The list of references is not complete and it is intended to as an introductory bibliography.

- Nuclear structure and BBN open questions: the cosmological ${ }^{7} \mathrm{Li}$ problem.

The chain of reactions known as BBN explains the sequence leading to the formation of the light elements. The predicted abundances are in good agreement with the experimental data, with the possible exception of ${ }^{7} \mathrm{Li} \mathrm{[1]}$. The theoretical predictions, starting from the neutron beta-decay until freeze-out, are based on nuclear reaction assumptions whose basic ingredients are thermal equilibrium and standard reaction rates and cross sections. The data show a nice agreement, between the calculated and observed abundances, except for ${ }^{7} \mathrm{Li}$. For this nucleus, the observed abundance is systematically smaller, by a factor 2 or so, than the calculated one. Naturally, one may try to fix the difference by using experimental cross sections, for reactions leading to ${ }^{7} \mathrm{Li}$. Among the possible explanations of the deficit in the abundance of ${ }^{7} \mathrm{Li}$, one may think of astrophysical mechanisms, like the trapping of primordial $\mathrm{Li}$ in the interior of stars $[5,6]$, of nuclear reactions involving resonances $[7,8]$, or just errors in the determination of the experimentally extracted values. While in some recent references, the dominance of astrophysical mechanisms has been advanced $[5,6]$, in some others, the nuclear reaction and nuclear structure sectors of the theory $[7,8]$ have been revisited to include resonances in the reaction channels involving $\mathrm{Be}$, in such a manner that the reduction in the abundance of ${ }^{7} \mathrm{Li}$ is just the result of decays which deplete the abundance of Be. This is, indeed, a very interesting problem, still unsolved, which may be further explored experimentally by performing measurements of nuclear reactions involving Be and its excited states, and theoretically, by performing nuclear structure calculation in the presence of 
resonances. Certainly, this can be achieved, for instance, by using shell model basis or other approximations, adapted to the handling of resonant single particle states.

- The mixing between sterile and active neutrinos: using cosmology to restrict the parameters of the mixing.

The analysis of the measurements performed by the Wilkinson Microwave Anisotropy Probe (WMAP) [1] and by Planck [2] have constrained rather accurately some relevant cosmological parameters, like the baryon to photon ratio and the effective number of neutrinos. These results are of importance at the time of making a comparison with theoretical models, particularly those which include the mixing between sterile and active neutrinos. One aspect which is particularly sensitive to the inclusion of sterile neutrinos in the neutrino spectrum is the determination of the neutrino occupation factors, relevant for the calculation of other cosmological observables, like light nuclei abundances. The theoretical models may accommodate one or more sterile neutrino species in interaction with the standard three mass eigenstates, such that the resulting spectrum, which will be dependent on extra mixing angles, may depart from the standard three-flavor scheme [9-11]. This is achieved in two steps, by solving the neutrino eigenvalue problem in presence of sterile neutrino, and then, by using the resulting spectrum in solving the evolution equation for an expanding Universe. The results of the calculations performed so far are rather interesting, since, once again, the calculations have opened the way to a very detailed comparison between observational data, like the ones of WMAP and Planck, with models which take into account different formulations of the cosmological framework by treating different degrees of freedom, as it is the case of models with more than one sterile neutrino species, varying cosmological parameters [12]. Thus, it would be very interesting to see, in future developments of the cosmological models, if the number of extra sterile neutrinos can be constrained and if their inclusion in leptonic mediated decays and reactions may explained for the data. Along this, one can discuss, for instance, the dependence of WMAP and Planck observables upon extra light- and heavy-mass sterile neutrinos.

- Neutrino masses from nuclear structure and cosmological considerations.

The nuclear neutrinoless double beta decay is perhaps the rarest event in nuclear and particle physics. It may proceed by massive Majorana neutrino exchange between nucleons in a nucleus, and because of its kinematics limitations it may be the unique way of determining the absolute scale of the neutrino mass spectrum. Naturally, because its leading contribution is a second order processes in the electro-weak Hamiltonian, the measurement of the half-life of a nuclear double beta decay emitter, which has a lower limit of the order of $10^{25}$ years $[3,4]$, is a real challenge to the imagination of experimental physicist $[13,14]$. In parallel to this, the value of the effective neutrino mass, relevant for the double beta decay, cannot be directly extracted, even if the decay is detected, without a precise control of the involved nuclear matrix elements, and, at least, without setting precise limits on each of the possible mechanisms, other than the mass one, which may contribute to the decay. The activity in this field is very intense, and will continue to be intense in the near future, because of the need to converge to reliable values of the relevant nuclear matrix elements, and because of the possibility of improvements from the experimental side, that is by the way of increasing the sensitivity to masses of the order of tens of meV. Smaller values of the neutrino mass may not be directly accessible by measurements, with the present techniques at least, but they will certainly point out to other scenarios, like super-symmetry (SUSY). In these models the light neutralino is a combination of super symmetric gauge-fermions and Higgs partners [15].

- Highly energetic neutrinos from galactic and extra galactic objects and their detection.

Highly energetic neutrinos may originate in micro-quasars, which are binary systems formed by a donor star and a compact object [17]. The accompanying collimated jets, where particles are accelerated to very high energies, are the source of high energy neutrinos. In this context, the effects of neutrino oscillations, on the neutrino fluxes coming from these astronomical objects, have been studied in the past years, and these studies have shown that the initial neutrino-flavor ratios are indeed very much affected by neutrino oscillations. The detection of neutrinos from micro-quasars is crucial to investigate the jet-composition and the particle acceleration processes inside the jet. Ice-cube type detectors [18] (or for the sake of the discussion, a cubic $\mathrm{Km}$ scale detector), may be sensitive to energies in the range $1-1000 \mathrm{TeV}$. The formalism, which consists of the expressions for the neutrino-flux as a function of astrophysical parameters, contains elements coming from the reaction sector, like the energy dependence of the proton spectrum, the neutrino sector, like the oscillation parameters and the interactions between the neutrino and the media. It is complemented by the calculations of the ratio between signals and noise, which is suppressed by neutrino oscillations [19]. However, if neutrinos of these sources are detected by a $\mathrm{km}$-scale detector, several constrains on the normalization constant, and consequently on astrophysical parameters, such as the total jet power, the compact object accretion rate, and the wind velocity, among others, can be obtained. The predicted reduction of the signal-to-noise ratio, produced by the inclusion of neutrino oscillation in the formalism, may substantially increase the time of observation. Certainly, all of these may need a very detailed exploration, from the theoretical side, and it can greatly contribute to a better understanding of the neutrino composition and its interactions.

\section{FINAL COMMENTS}

Neutrino physics is an extremely rich branch of physics, with intense links between nuclear physics, particle physics, astronomy, astro-particle physics and cosmology. It is a very rich source of motivation for studies in the domain of few $\mathrm{meV}$ to hundreds of $\mathrm{TeV}$. It may be the only possible way to demonstrate the need of theories beyond the standard model of electroweak interactions and to pave the way to highly hierarchical models, like supersymmetry. The atomic nucleus is the primary laboratory, since it may signal the existence of extremely rare decays from where one can extract information about the neutrino. In 
addition, since they share a fair deal of common grounds, the search for leptonic cold dark-matter is strongly tied up to nuclear processes, as it will be the case if the seasonal modulation of cold dark matter signals is confirmed [16]. Thus, we have here a cornerstone in neutrino studies which will eventually open the way to a deeper understanding of the structure of the Universe, as it can be easily grasped from the consequences of a direct measurement of these exotic processes. Another potential line of research is the complementarity existing between low and high energy phenomena involving neutrinos. One can thus investigate the connection between high energy observables extracted from LHC data (like ATLAS and CMS data on heavy neutrinos) and low energy decay processes, like neutrinoless double beta decay to eventually set-up limits on the left-right extensions of the Standard Model.

The Universe is the ultimate tool to enlarge the knowledge on neutrinos, both by testing cosmology and by observing highly energetic processes with the participation of neutrinos. The complexity of all of this may be overcome by the feeling that it is part of the beauty of the landscape which may be around the corner. Thus, the neutrino could be the tiny messenger (piccolo postino) revealing it.

\section{REFERENCES}

1. Larson D, Dunkley J, Hinshaw G, Komatsu E, Nolta MR, Bennett CL, et al. Seven-year wilkinson microwave anisotropy probe (WMAP) observations: power spectra and WMAP-derived parameters. ApJS. (2011) 192:16. doi: 10.1088/0067-0049/192/2/16

2. Ade PAR, Aghanim N, Armitage-Caplan C, Arnaud M, Ashdown M, AtrioBarandela F, et al. Planck 2013 results. XVI. Cosmological parameters. arXiv:1303.5076 [astro-ph.CO] (2013).

3. Agostini M, Allardt M, Andreotti E, Bakalyarov AM, Balata M, Barabanov I, et al. Results on neutrinoless double- $\beta$ decay of Ge76 from Phase I of the GERDA Experiment. Phys Rev Lett. (2013) 111:122503. doi: 10.1103/ PhysRevLett.111.122503

4. Albert JB, Auty DJ, Barbeau PS, Beauchamp E, Beck D, Belov V, et al. Search for Majorana neutrinos with the first two years of EXO-200 data. arXiv:1402.6956 [nucl-ex] (2014)

5. Richard O, Michaud G, Richer J. Implications of WMAP observations on Li abundance and stellar evolution models. Astrophys J. (2005) 619:538-548. doi: $10.1086 / 426470$

6. Meléndez J, Casagrande L, Ramírez I, Asplund M, Schuster WJ. Observational evidence for a broken Li Spite plateau and mass-dependent Li depletion. Astron Astrophys. (2010) 515:1-7. doi: 10.1051/0004-6361/ 200913047
7. Kirsebom OS, Davids B. One fewer solution to the cosmological lithium problem. Phys Rev C. (2011) 84:058801. doi: 10.1103/PhysRevC.84.058801

8. Broggini C, Canton L, Fiorentini G, Villante FL. The cosmological 7Li problem from a nuclear physics perspective. arXiv:1202.5232 [astro-ph.CO] (2012).

9. Burenin RA, Vikhlinin AA. Cosmological parameters constraints from galaxy cluster mass function measurements in combination with other cosmological data. arXiv:1202.2889 [astro-ph.CO] (2012). doi: 10.1134/S10637737 12060011

10. Ichikawa K, Sekiguchi T, Takahashi T. Probing the effective number of neutrino species with the cosmic microwave background. Phys Rev D. (2008) 78:083526. doi: 10.1103/PhysRevD.78.083526

11. Acero MA, Lesgourgues J. Cosmological constraints on a light nonthermal sterile neutrino. Phys Rev D. (2009) 79:045026. doi: 10.1103/PhysRevD.79. 045026

12. Kishimoto CT, Fuller GM, Smith CJ. Coherent active-sterile neutrino flavor transformation in the early universe. Phys Rev Lett. (2006) 97:141301. doi: 10.1103/PhysRevLett.97.141301

13. Suhonen J, Civitarese O. Weak-interaction and nuclear-structure aspects of nuclear double beta decay. Phys Rep. (1998) 300:123. doi: 10.1016/S0370-1573 (97)00087-2

14. Ejiri H. Nuclear spin isospin responses for low-energy neutrinos. Phys Rep. (2000) 338:265. doi: 10.1016/S0370-1573(00)00044-2

15. Vergados JD. The Neutrinoless double beta decay from a modern perspective. Phys Rep. (2002) 361:1. doi: 10.1016/S0370-1573(01)00068-0

16. Bernabei R, Belli P, Cappella F, Cerulli R, Dai CJ, D’Angelo A, et al. First results from DAMA/LIBRA and the combined results with DAMA/NaI. Eur Phys J C. (2010) 56:333-355. doi: 10.1140/epjc/s10052-008-0662-y

17. Aharonian F, Akhperjanian AG, Aye K-M, Bazer-Bachi AR, Beilicke M, Benbow W, et al. Discovery of very high energy gamma rays associated with an X-ray binary. Science (2005) 309:746-749. doi: 10.1126/science.1113764

18. IceCube. Available online at: http://icecube.wisc.edu/

19. Costantini ML, Vissani F. Expected neutrino signal from supernova remnant RX J1713.7-3946 and flavor oscillations. Astropart Phys. (2005) 23:477-485. doi: $10.1016 /$ j.astropartphys.2005.03.003

Conflict of Interest Statement: The author declares that the research was conducted in the absence of any commercial or financial relationships that could be construed as a potential conflict of interest.

Received: 24 September 2013; accepted: 12 May 2014; published online: 02 June 2014. Citation: Civitarese O (2014) Neutrino-related questions in nuclear and astroparticle physics. Front. Physics 2:35. doi: 10.3389/fphy.2014.00035

This article was submitted to High-Energy and Astroparticle Physics, a section of the journal Frontiers in Physics.

Copyright (c) 2014 Civitarese. This is an open-access article distributed under the terms of the Creative Commons Attribution License (CC BY). The use, distribution or reproduction in other forums is permitted, provided the original author(s) or licensor are credited and that the original publication in this journal is cited, in accordance with accepted academic practice. No use, distribution or reproduction is permitted which does not comply with these terms. 